\title{
Extrasynaptic release of serotonin affects the social dynamics of leeches
}

\author{
Enrique Hernández-Lemus* \\ Computational Genomics Department, National Institute of Genomic Medicine, México City, Mexico \\ *Correspondence: ehernandez@inmegen.gob.mx
}

\section{A commentary on}

The dynamics of group formation among leeches

by Bisson, G., Bianconi, G., and Torre, V. (2012). Front. Physiol. 3:133. doi: 10.3389/ fphys.2012.00133

In order to attain significative advance in almost any area in modern-day scientific research, it is often necessary to think outof-the-box. That is, if we want to catch something fresh and new, we need to abandon traditional paradigms and preconceived ways of reasoning. In this sense, the paper by Bisson et al. (2012) in this special issue results quite enlightening, for it seems that, their way of thinking out of the box is based on their ability to think inside many boxes at a time. In this paper, the authors discuss about group formation in leeches, and the way that extrasynaptic neuromodulation affects this phenomenon. Group formation is shown to be facilitated by body injection of serotonin (5-HT). It is also mentioned how the level of endogenous 5-HT is elevated in leeches belonging to a large group. To test the role of 5-HT neuromodulation in group formation, leeches were injected with 5-HT antagonists that prevented them from joining a large group. Sensilla ablation and supraesophageal ganglion disconnection also prevented group formation. It is discussed the role that neurochemicals may have on social decision making in leeches, resembling the case in human interactions. Interesting parallels were thus sketched between social group formation in leeches and in humans. For instance, the authors revealed the presence of social reinforcement dynamics as a feature of group formation. Social reinforcement is known to play an important role in human social interactions (Cattuto et al., 2009; Stehlé et al., 2010). It is also shown that before socializing on a permanent basis, leeches first "get acquainted with each other" as the authors argue in the beginning of the subsection Properties of group formation, recalling that groups formed in the first hour are not stable.

The findings and conclusions of this paper are thus very appealing and intriguing. However, no less riveting result the methods used to reach such conclusions. Apart from the obvious display of ability in the methods of experimental neurobiology; the group, headed by Professor Vincent Torre, embarked upon a research project that combines ethology (by studying leech behavior), social network dynamics (e.g., reinforcement dynamics), imaging and image processing (image acquisition, discretization techniques), computational geometry (contour-tracing and polygon-filling algorithms), mathematical optimization (constrained quadratic programming), and an overall view from the standpoint of complex systems science; all of this, without abandoning their neurobiological foundations.

The scope of studies of this kind, as well as their outreach possibilities, is large. Such disparate areas of research as social network epidemiology (Christakis and Fowler, 2008, 2012), flocking and swarming (Miramontes and De Souza, 2008; Aldana et al., 2009; Nagy et al., 2010), collective neural computation (Hopfield, 1982; Colorni et al., 1992), and of course, physiology and neuroscience, may be influenced by the ideas and methods combined in this study. Last but not least we may mention another feature of this paper that, while it should be a rule on scientific inquiry, it results somehow dismissed in many instances nowadays, likely due to publication pressure. We refer to the role that alternative hypothesis testing should have in research. In order to prove their point about the role that 5-HT may have in the dynamics of group formation in leeches, the authors injected leeches with both, the neurotransmitter and its antagonists, tested the medium for chemotaxis, ablated the Sensilla and disconnected the supraesophageal ganglion to physically block the effects of 5-HT. They also probed about the influence of illumination and seasonal variability for the possible impact on their results. Overall: a gripping, novel-yet somehow risky-analysis, on a quite outstanding series of experimental results that seem to suggest a non-trivial relation between extrasynaptic signaling and social behavior.

\section{REFERENCES}

Aldana, M., Larralde, H., and Vázquez, B. (2009). On the emergence of collective order in swarming systems: a recent debate. Int. J. Mod. Phys. B 23, 3661-3685.

Bisson, G., Bianconi, G., and Torre, V. (2012). The dynamics of group formation among leeches. Front. Physiol. 3:133. doi: 10.3389/fphys.2012.00133

Cattuto, C., Barrat, A., Baldassarri, A., Schehr, G., and Loreto, V. (2009). Collective dynamics of social annotation. Proc. Natl. Acad. Sci. U.S.A. 106, 10511-10515.

Christakis, N. A., and Fowler, J. H. (2008). The collective dynamics of smoking in a large social network. $N$. Engl. J. Med. 358, 2249-2258.

Christakis, N. A., and Fowler, J. H. (2012). Social network sensors for early detection of contagious outbreaks. PLoS ONE 5, e12948. doi: 10.1371/journal. pone.0012948

Colorni, A., Dorigo, M., and Maniezzo, V. (1992). "Distributed optimization by ant colonies," in Toward a Practice of Autonomous Systems: Proceedings of the First European Conference on Artificial Life, F. eds J. Varela and P. Bourgine (Cambridge, MA: MIT Press/ Bradford Books), 1341-42.

Hopfield, J. J. (1982). Neural networks and physical systems with emergent collective computational abilities. Proc. Natl. Acad. Sci. U.S.A. 79, 2554-2558.

Miramontes, O., and De Souza, O. (2008). Individual basis for collective behaviour in the termite, Cornitermes cumulans. J. Insect Sci. 8, 1-22.

Nagy, M., Ákos, Z., Biro, D., and Vicsek, T. (2010). Hierarchical group dynamics in pigeon flocks. Nature 464, 890-893.

Stehlé, J., Barrat, A., and Bianconi, G. (2010). Dynamical and bursty interactions in social networks. Phys. Rev. E 81, 035101.

Received: 02 May 2012; accepted: 06 May 2012; published online: 28 May 2012.

Citation: Hernández-Lemus E (2012) Extrasynaptic release of serotonin affects the social dynamics of leeches. Front. Physio. 3:158. doi: 10.3389/fphys.2012.00158

This article was submitted to Frontiers in Membrane Physiology and Biophysics, a specialty of Frontiers in Physiology.

Copyright (@) 2012 Hernández-Lemus. This is an open-access article distributed under the terms of the Creative Commons Attribution Non Commercial License, which permits noncommercial use, distribution, and reproduction in other forums, provided the original authors and source are credited. 